\section{Topische Behandlung der Akne mit Azelainsäure}

Die Akne der erwachsenen Frau (Acne tarda) tritt entweder erstmals nach dem 25. Lebensjahr auf (late-onset-acne) oder persistiert von der Pubertät an (bei 59-82\% der betroffenen Frauen). Sie betrifft bei erwachsenen Frauen vorwiegend das Gesicht (untere Wangenpartien, seitlicher Unterkieferrand, Perioralregion, Kinn = U-Zone) - im Gegensatz zur Pubertätsakne, bei der eine Lokalisation in der T-Zone des Gesichts (Stirn, Nase, obere Wangenpartien) überwiegt und eine Beteiligung des oberen Stammbereiches häufig ist.

Ein wichtiger Faktor in der Pathogenese der Acne tarda ist die erhöhte Talgdrüsenaktivität (Seborrhö) mit Synthese proinflammatorischer Lipide, aber auch eine gestörte follikuläre Differenzierung, verstärkte Verhornung (Hyperkeratinisierung) sowie Entzündungsreaktionen.

Zusätzlich wird Akne stark durch hormonelle Schwankungen während des Zyklus beeinflusst, weshalb bei etwa $70 \%$ der Frauen eine Verschlechterung von Akneläsionen während der Menstruation zu beobachten ist. Studien deuten zudem auf einen Zusammenhang zwischen der Talgproduktion und der Dichte der Besiedelung mit Propionibacterium acnes hin [Ramos-e-Silva M et al. Br J Dermatol 2015].

Für die topische Behandlung stehen laut aktueller S2-Leitlinie der Deutschen Dermatologischen Gesellschaft verschiedene Wirkstoffe zur Verfügung. Azelainsäure (Skinoren ${ }^{\circledR}$ ) weist sowohl antiinflammatorische als auch komedolytische, antikomedogene und antimikrobielle Eigenschaften auf [Mastrofrancesco A et al. Exp Dermatol 2010]. Es wird bei leichten bis mittelschweren Akneformen allein oder in Kombination mit Benzoylperoxid, topischen Antibiotika und Retinoiden sowie systemischen Antibiotika empfohlen. Auch eine Kombination mit systemischen, hormonellen EstrogenAntiandrogen-Kombinationen ist möglich. Azelainsäure ist als Monotherapie auch in der Schwangerschaft mit Bedacht anwendbar [Nast A et al. J Dtsch Dermatol Ges 2010].

Nach Informationen von Jenapharm

Wirkkomplex aus Bitterstoffen

\title{
Natürliche Anti-Aging-Pflege
}

Ein Team um Prof. Christoph Schempp und Ute Wölfle, Uniklinik Freiburg, entdeckten Bitterstoffrezeptoren der Haut, entschlüsselte deren Wirkmechanismus und machten diese für die Hautpflege nutzbar. Führt man der Haut Bitterstoffe zu, so die Entdeckung der Forscher, wird die Bildung von Lipiden und Schutzproteinen angeregt, der Hautstoffwechsel stimuliert und die Hautbarriere gestärkt. „Immer wenn die Pflege auf die Haut aufgetragen wird, entsteht eine Symbiose und es startet ein Prozess, der gut für die Haut ist. Und das Ganze auf natürlicher Basis mit den Extrakten von sorgfältig ausgewählten Heilpflanzen“, so Schempp, Gründer von Velan Skincare. Das Ergebnis: sechs eigens konzipierte Pflegeprodukte für "ruhige“, „reine“ und „straffe“ Haut. Herzstück ist dabei der Extrakt aus dem gelben Enzian in Bio-Qualität.

Velan-Ruhige-Haut-Gesichts-Creme und Körper-Balsam enthalten keine Duftstoffe und wurden für empfindliche, zu Juckreiz neigende Haut entwickelt.
Das Süßholz und die in der Süßholzwurzel enthaltene Glycyrrhetinsäure beruhigen empfindliche, zu Rötungen neigende Haut. Bio-Granatapfelsamenöl und Sheabutter unterstützen die Regeneration der Hautbarriere.

Die Velan-Reine-Haut-Peeling-Maske spendet bei empfindlicher Haut milde Reinigung und Pflege in einem Schritt. DasVelan-Reine-Haut-Akut-Gel bekämpft Unreinheiten mit pflanzlichen Wirkstoffen. Weidenrinde enthält Polyphenole (Gerbstoffe, Flavonoide), Salicylsäure und Salicin (Weidenbitter).

Für die Erhaltung einer straffen Haut wurde das Gesichtsserum und eine Pflegelotion entwickelt. Auf Basis von Schisandraextrakt und Luteolin wirken beide Produkte antioxidativ, schützen das Bindegewebe der Haut und glätten die Haut durch regenerationsfördernde Wirkstoffe und natürliche Feuchthaltefaktoren.

red

Nach Informationen von Velan Skincare

\section{Endovenöse Lasertherapie}

\section{Behandlung von insuffizienten Venen}

Das ELVeS-Radial ${ }^{\mathrm{TM}}$-Laserverfahren ermöglicht eine schonende und nebenwirkungsarme Behandlung von Insuffizienz der Vena saphena magna, der Vena saphena parva und insbesondere von Perforansvenen und Seitenästen. Da die ELVeSRadial-2ring ${ }^{\mathrm{Tm}}$-Faser zweiphasig abstrahlt, weisen die beiden Lichtringe eine geringere Energiedichte auf. Die Abstrahlung in der Vene ist hierbei homogener als bei einer einphasig abstrahlenden Faser. Dadurch wird die Vene präzise von innen verschlossen. Das Risiko einer Perforation der Venenwand wird so minimiert.

Die ELVeS-Radial-slim ${ }^{\mathrm{m}}$-Laserfaser wurde für besonders dünne Venen entwickelt. Mit ihr können auch kompliziertere anatomische Strukturen und sogar Stripping-Rezidive problemlos behandelt werden. Der Zugang der atraumatischen Faserspitze in die Vene erfolgt über einen Katheter und unter Einsatz von Lokalan- ästhesie, femoralem Nervenblock oder einem leichten Beruhigungsmittel. Überwacht wird der Zugang und die Platzierung der Faser über Ultraschall. Nebenwirkungen treten bei einer lasergestützten Varizenbehandlung deutlich seltener auf.

Durch die Fusion ${ }^{\oplus}$-Technologie der biolitec $^{\circledast}$ wird während der Behandlung höchste Sicherheit gewährleistet. Die Faserspitze aus Quarz wird mit dem Lichtleiter sicher und fest verschweißt und nicht einfach nur verklebt. Alle Lichtleiter - auch die zur minimalinvasiven Behandlung von Hämorrhoiden, Fisteln, Myomen oder benignen Prostataveränderungen - sind mit dieser Technologie ausgestattet.

Weitere Informationen erhalten Sie über die Webseite www.biolitec.de. red

Nach Informationen von biolitec biomedical technology 